\title{
Sequential afatinib and osimertinib in patients with EGFR mutation-positive non-small-cell lung cancer: final analysis of the GioTag study
}

\author{
Maximilian J Hochmair*, (D), Alessandro Morabito² (D), Desiree Hao ${ }^{3}$, Cheng-Ta Yang ${ }^{4}$, Ross \\ A Soo ${ }^{5}$, James C-H Yang ${ }^{6}$, Rasim Gucalp7 , Balazs Halmos , Angela Märten ${ }^{8}$ \& Tanja Cufer ${ }^{9}$ \\ ${ }^{1}$ Department of Respiratory \& Critical Care Medicine, Karl Landsteiner Institute of Lung Research \& Pulmonary Oncology, \\ Krankenhaus Nord, 3500 Vienna, Austria \\ ${ }^{2}$ Thoracic Medical Oncology, Istituto Nazionale Tumori, "Fondazione G. Pascale"-IRCCS, 80131 Napoli, Italy \\ ${ }^{3}$ Tom Baker Cancer Center, Cummings School of Medicine, University of Calgary, Calgary, AB T2N 4N1, Canada \\ ${ }^{4}$ Department of Thoracic Medicine, Chang Gung Memorial Hospital, 333 Taoyuan, Taiwan \\ ${ }^{5}$ Department of Haematology-Oncology, National University Hospital, Singapore \\ ${ }^{6}$ Department of Oncology, National Taiwan University Hospital \& National Taiwan University Cancer Center, Taipei, Taiwan \\ ${ }^{7}$ Department of Oncology, Montefiore/Albert Einstein Cancer Center, Bronx, NY 10461, USA \\ ${ }^{8}$ Boehringer Ingelheim International GmbH, 55216 Ingelheim am Rhein, Germany \\ ${ }^{9}$ University Clinic Golnik, University of Ljubljana, 4204 Ljubljana, Slovenia \\ *Author for correspondence: Tel.: +43 1910604 1240; maximilian.hochmair@wienkav.at
}

Aim: Final overall survival (OS) and time on treatment analysis of patients with EGFR mutation-positive non-small-cell lung cancer (NSCLC) who received sequential afatinib and osimertinib. Patients \& methods: Patients $(n=203)$ had T790M-positive disease following first-line afatinib and started osimertinib treatment $\geq 10$ months before data entry. Primary outcome was time on treatment; OS analysis was exploratory. Results: Median time on treatment with afatinib and osimertinib was 27.7 months (90\% Cl: 26.7-29.9). Median OS was 37.6 months (90\% Cl: 35.5-41.3); median OS was 41.6 and 44.8 months in Del19-positive patients and Asian patients, respectively. Conclusion: In real-world clinical practice, sequential afatinib and osimertinib was associated with encouraging outcomes in patients with EGFR mutation-positive NSCLC, especially in Del19-positive patients and Asian patients.

Clinical Trial Registration: NCT03370770 (ClinicalTrials.gov)

First draft submitted: 22 July 2020; Accepted for publication: 13 August 2020; Published online: 28 August 2020

Keywords: afatinib • EGFR • NSCLC • osimertinib

Three generations of epidermal growth factor receptor (EGFR) tyrosine kinase inhibitors (TKIs) are now approved in the first-line setting for patients with EGFR mutation-positive non-small-cell lung cancer (NSCLC): the firstgeneration reversible TKIs, erlotinib and gefitinib; the second-generation irreversible ErbB family blockers, afatinib and dacomitinib; and the third-generation EGFR TKI, osimertinib [1-5].

In randomized clinical trials, the second- and third-generation EGFR TKIs have significantly improved progression-free survival versus first-generation TKIs in first-line treatment of EGFR mutation-positive NSCLC [68]. Exploratory analysis of the ARCHER-1050 trial indicated that dacomitinib was associated with improved overall survival (OS) versus gefitinib, and LUX-Lung 7 showed a trend toward OS benefit with afatinib [9,10]. Recent data from the FLAURA Phase III trial demonstrated significantly prolonged OS with first-line osimertinib compared with the first-generation EGFR TKIs (gefitinib or erlotinib) in patients with EGFR mutation-positive NSCLC [11]. However, as acquired resistance to first-line EGFR TKI therapy is inevitable, the availability of subsequent treatment options following disease progression is a key consideration when assessing therapeutic choices.

Emergence of the T790M mutation in exon 20 of EGFR is the predominant molecular resistance mechanism to gefitinib, erlotinib and afatinib. This mutation presents in approximately $50-73 \%$ of tumors at the time of 
acquired resistance, with the likelihood being highest in patients with Del19-positive disease [12-16]. Osimertinib has demonstrated impressive activity in T790M-positive patients [17]. In contrast, targeted therapy options following first-line osimertinib treatment remain limited due to the heterogeneity of osimertinib resistance mechanisms, which are still not fully understood $[18,19]$. Chemotherapy is often the only option for patients who progress on osimertinib treatment in everyday clinical practice.

It has therefore been suggested that, at least in some patients, reserving osimertinib as a second-line therapy option may maximize time on targeted treatment and defer the need for more toxic chemotherapy regimens. The GioTag study was a global, observational, multicenter study designed to assess outcomes in EGFR TKI-naive patients with EGFR mutation-positive (Del19/L858R) NSCLC who received sequential afatinib and osimertinib treatment in a real-world clinical practice setting [20,21]. Importantly, for real-world clinical practice, the study included elderly patients and those with poor prognostic characteristics (Eastern Cooperative Oncology Group performance status [ECOG PS] $\geq 2$ or stable brain metastases) who are often under-represented in or excluded from randomized clinical trials.

At the initial and updated analyses (May 2018 and April 2019, respectively), results were encouraging, particularly for Del19-positive patients and Asian patients [20,21]. Here, we report findings from the final analysis, including updated time on treatment and OS data.

\section{Materials \& methods}

Study design \& patients

The design of the GioTag study has been described previously [20,21]. In brief, GioTag was a global, observational study conducted across ten countries (Austria, Canada, Israel, Italy, Japan, Singapore, Slovenia, Spain, Taiwan and the USA; NCT03370770). Data were collected between December 2017 and December 2019 for patients with EGFR mutation-positive (Del19 and L858R) NSCLC who had T790M-positive disease after first-line afatinib and subsequently received osimertinib. To limit selection bias, each participating center assessed the health records of a maximum of 15 consecutive patients. All patients must have initiated osimertinib $\geq 10$ months prior to enrollment to avoid early censoring and ensure mature data. Data were collected directly from sites via manual medical chart review $(\mathrm{n}=77 ; 38 \%)$ or from electronic health records $(\mathrm{n}=126 ; 62 \%)$ supplied by Cardinal Health $(\mathrm{OH}, \mathrm{USA})$. Verification of source data were undertaken for $30 \%$ of patients. Informed consent was provided where required.

\section{Outcomes \& assessments}

The primary outcome was time on treatment, defined as the time from the first dose of afatinib to that of the last dose of osimertinib or death. The OS analysis was exploratory and was defined as time from start of afatinib treatment to death.

\section{Statistical analysis}

Data cut-off for this final analysis was 28 November 2019 and data for all enrolled patients were included. Time on treatment and OS were estimated using the Kaplan-Meier method; for patients still on treatment, time on treatment was censored at the date of data collection.

\section{Results}

Baseline demographics and characteristics of the 204 patients included in the analysis have been described previously [20,21]. The GioTag population reflected real-world clinical practice and included patients with ECOG PS $\geq 2$ $(15.2 \%)$ and those with CNS metastases (10.3\%), in addition to the usual patient population included in clinical trials. Patients were predominantly Caucasian (58.8\%) but also included Asian (24.5\%) and African-American $(8.8 \%)$ patients. At the start of afatinib treatment, $73.5 \%$ of patients had a Del19 mutation and $26.0 \%$ had the L858R mutation. One patient had both Del19 and L858R.

Most patients received the approved starting doses of afatinib $(40 \mathrm{mg} /$ day; $83.7 \%)$ and osimertinib $(80 \mathrm{mg} /$ day; $98.0 \%)$. One patient was excluded from the analysis due to reports of conflicting data. At the time of this final analysis (December 2019), 120 (59.1\%) patients had died, 31 (15.3\%) were lost to follow-up and 52 (25.6\%) were alive; of these 52, 29 remained on osimertinib treatment and 11 had discontinued osimertinib treatment.

After a median follow-up of 33.9 months, the median time on treatment for sequential afatinib and osimertinib was 27.7 months (90\% CI: 26.7-29.9; Figure 1A). For Asian patients $(\mathrm{n}=50)$, median time on treatment was 37.1 months (90\% CI: 28.1-40.3) and in patients with Del19-positive tumors ( $\mathrm{n}=149)$, median time on treatment 
(A)

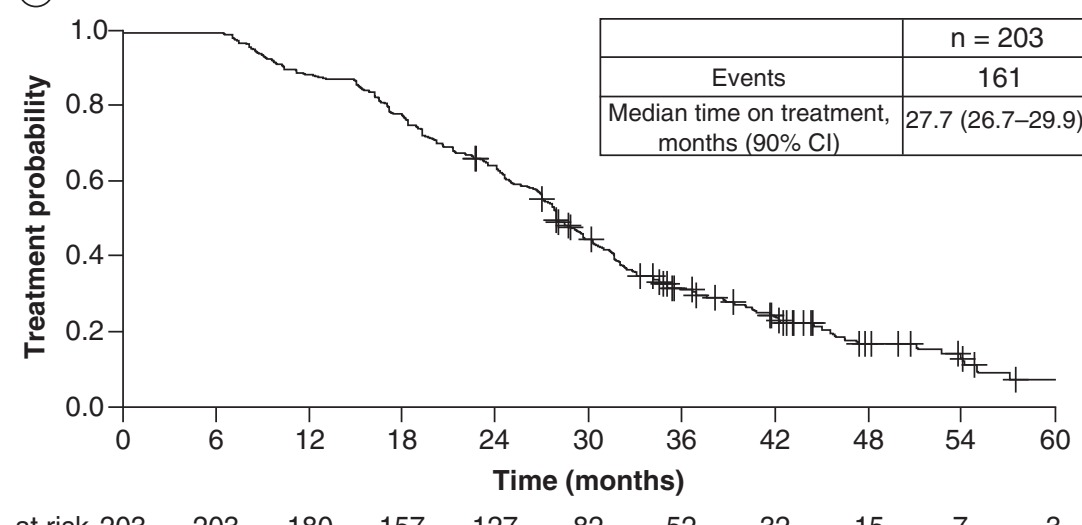

$\begin{array}{lllllllllll}\text { No. at risk } 203 & 203 & 180 & 157 & 127 & 82 & 52 & 32 & 15 & 7 & 3\end{array}$

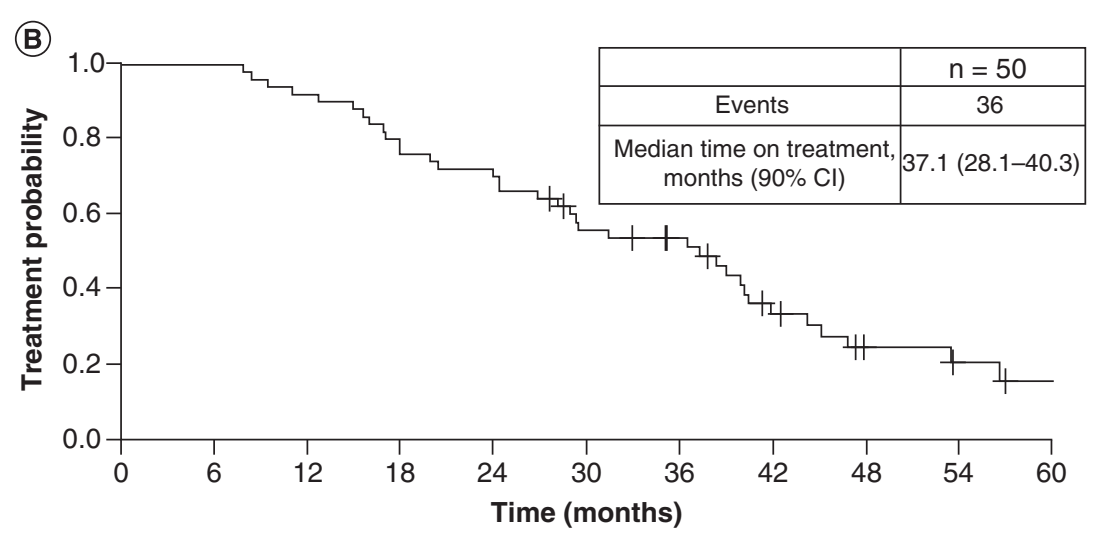

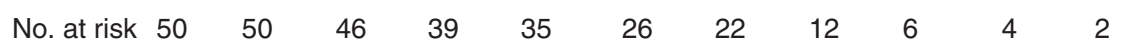

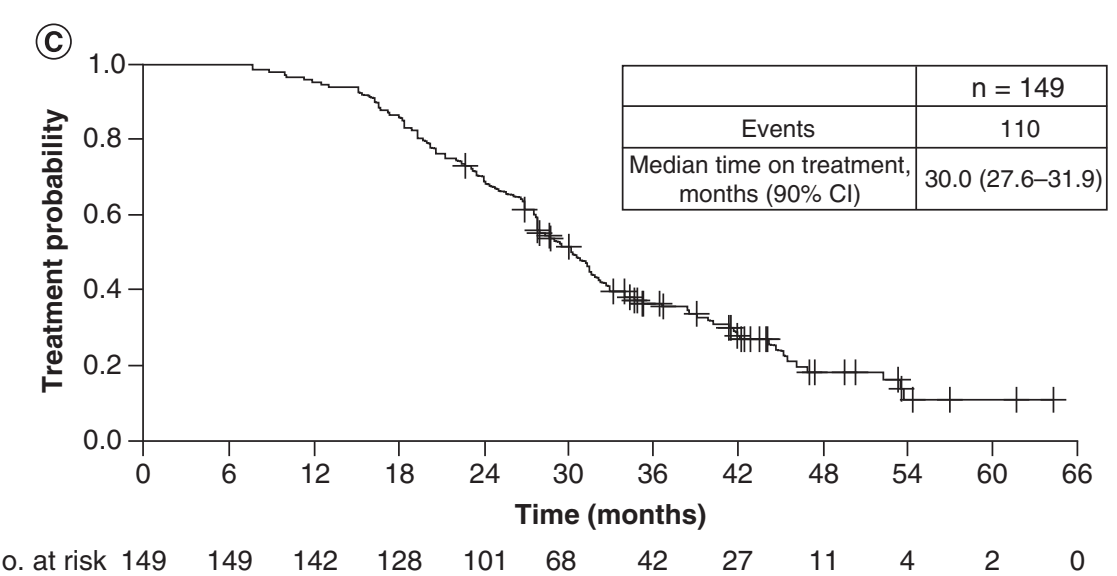

Figure 1. Time on treatment with sequential afatinib and osimertinib. (A) All patients; (B) Asian patients; and (C) patients with Del19-positive tumors.

was 30.0 months (90\% CI: 27.6-31.9) (Table $1 \&$ Figure 1). In the 31 Asian patients with Del19-positive disease, median time on treatment was 40.0 months (90\% CI: 36.4-45.0). Clinical benefit was also consistent across patient subgroups often excluded from clinical trials: median time on treatment was 22.2 months in patients with brain metastases, 27.3 months in patients aged $\geq 65$ years and 22.2 months in those with ECOG PS $\geq 2$ (Table 1).

As reported previously, overall median time on afatinib was 11.9 months (90\% CI: 10.9-12.2) [20]. Median time on osimertinib treatment was 15.6 months (90\% CI: 13.6-17.1) overall, 18.9 months (90\% CI: 13.6-23.3) in Asian patients and 16.5 months (90\% CI: 14.9-17.9) in patients with Del19-positive tumors.

Overall median OS was 37.6 months (90\% CI: 35.5-41.3) with a 2-year survival rate of $80 \%$ (Figure $2 \mathrm{~A}$ ). 
Short Communication Hochmair, Morabito, Hao et al.

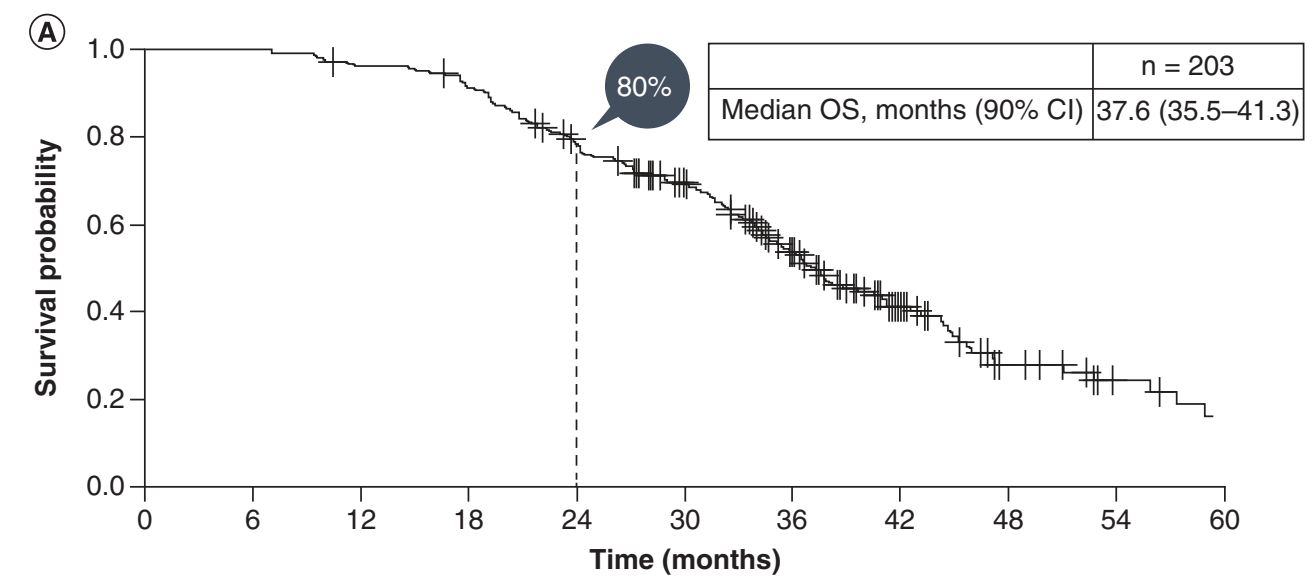

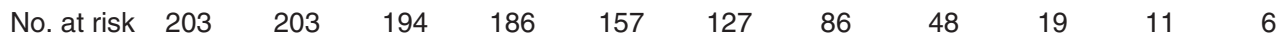

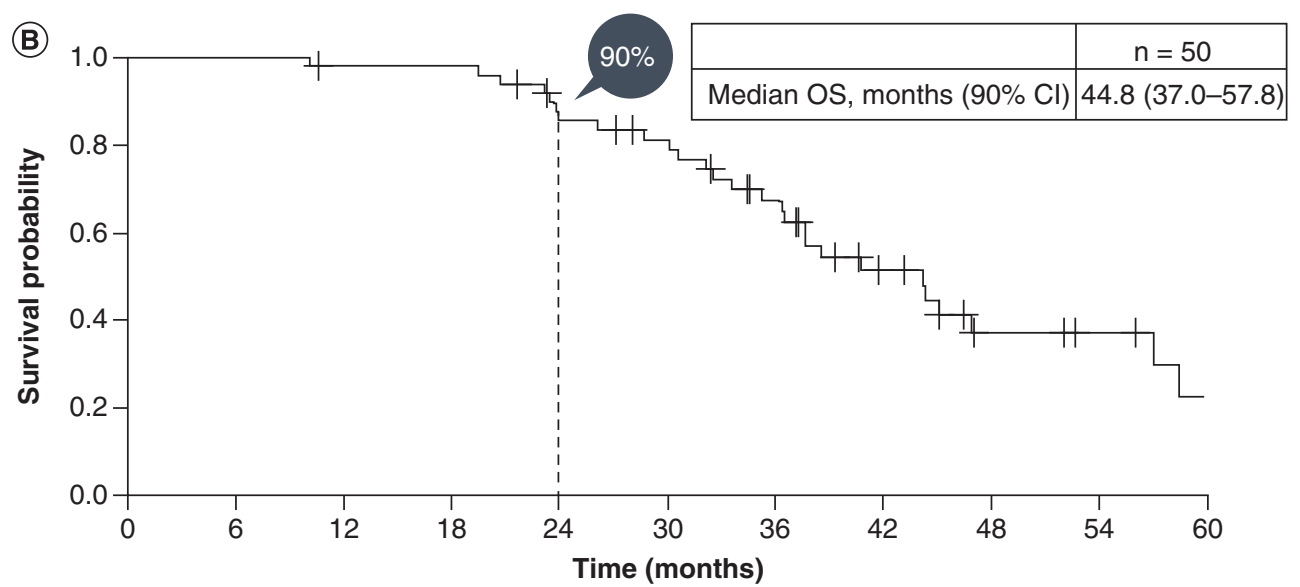

$\begin{array}{llllllllllll}\text { No. at risk } & 50 & 50 & 48 & 48 & 42 & 36 & 27 & 17 & 7 & 6 & 3\end{array}$

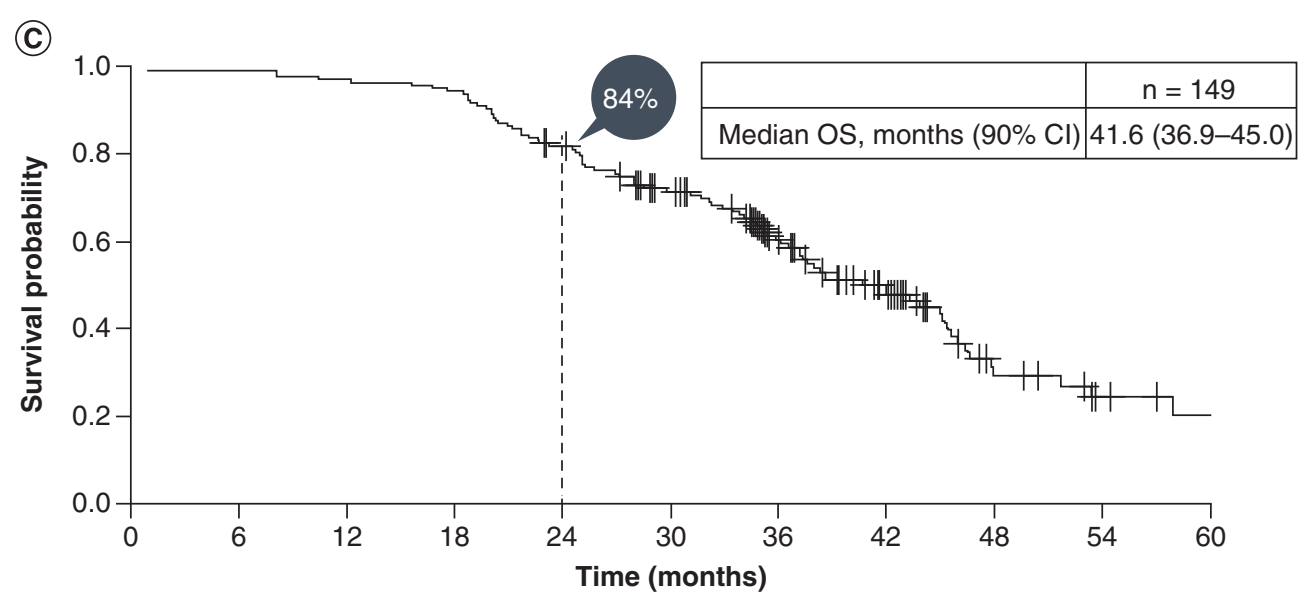

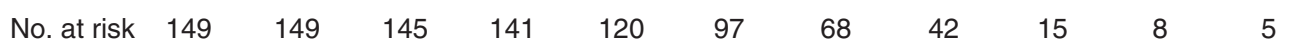

Figure 2. Overall survival in patients treated with sequential afatinib and osimertinib. (A) All patients; (B) Asian patients; and (C) patients with Del19-positive tumors.

OS: Overall survival. 


\begin{tabular}{|c|c|c|}
\hline Baseline demographic/disease characteristic & Median time on treatment $(90 \% \mathrm{Cl})$, months & Median OS ( $90 \% \mathrm{Cl})$, months \\
\hline Overall population & $27.7(26.7-29.9)$ & $37.6(35.5-41.3)$ \\
\hline Non-Asian $(n=137)$ & $27.6(26.3-29.3)$ & $36.7(34.4-41.6)$ \\
\hline Asian $(n=50)$ & $37.1(28.1-40.3)$ & $44.8(37.0-57.8)$ \\
\hline \multicolumn{3}{|l|}{ Age at start of afatinib (years) } \\
\hline$\geq 65$ years $(n=71)$ & $27.3(20.4-31.3)$ & $36.9(33.0-44.8)$ \\
\hline \multicolumn{3}{|l|}{ EGFR mutation at start of afatinib } \\
\hline Del19 $(n=149)$ & $30.0(27.6-31.9)$ & $41.6(36.9-45.0)$ \\
\hline L858R $(n=53)$ & $19.1(16.8-26.3)$ & $33.0(29.8-37.0)$ \\
\hline \multicolumn{3}{|l|}{ Presence of brain metastases } \\
\hline $0 / 1(n=152)$ & $30.0(28.1-31.7)$ & $41.0(37.6-45.0)$ \\
\hline$\geq 2(n=31)$ & $22.2(16.0-26.5)$ & $32.0(24.5-34.5)$ \\
\hline
\end{tabular}

Median OS was 44.8 months (90\% CI: 37.0-57.8) in Asian patients and 41.6 months (90\% CI: 36.9-45.0) in patients with Del19-positive disease (Figure 2); in Asian patients with Del19-positive disease, OS was 45.7 months (90\% CI: 38.2-57.8). Median OS was consistent in patients with poor prognostic characteristics: 31.0 months in patients with brain metastases, 36.9 months in patients aged $\geq 65$ years and 32.0 months in those with ECOG PS $\geq 2$ (Table 1). Median time from discontinuation of osimertinib treatment to death was 5.6 months $(90 \% \mathrm{CI}$ : 4.3-8.0).

For the 168 patients who received the recommended starting dose of afatinib $(40 \mathrm{mg})$, median time on treatment and OS were 27.7 months (90\% CI: 26.7-29.9) and 38.0 months (90\% CI: 35.9-41.3), respectively. Median time on treatment and OS were 38.2 months (90\% CI: 28.9-40.3) and 44.8 months (90\% CI: $38.2-57.8$ ) in Asian patients and 29.9 months (90\% CI: 27.6-32.7) and 40.3 months (90\% CI: 36.8-44.8) in those with Del19-positive disease, respectively. In the 29 Asian patients with Del19-positive disease who started on afatinib $40 \mathrm{mg}$, median time on treatment and OS were 40.0 months (90\% CI: 36.4-46.7) and 45.0 months (90\% CI: 38.2-57.8), respectively.

\section{Discussion}

These final results of the GioTag study further demonstrate that sequential afatinib and osimertinib treatment is a feasible and effective therapeutic strategy in a broad, real-world population of patients with EGFR mutationpositive NSCLC who acquired T790M, confirming results from the previous analyses [20,21]. Overall, median time on sequential afatinib and osimertinib treatment was 27.7 months for this patient population, consistent with the findings of the primary and interim analyses of the GioTag study (median times on treatment of 27.6 and 28.1 months, respectively) [20,21]. The OS data reported here represent the most mature analysis of OS with sequential afatinib and osimertinib to date. Particularly favorable outcomes were seen in patients with Del19positive disease and Asian patients, with prolonged median time on treatment and a median OS of over 3.5 years reported for both subgroups. Across the overall population and patient subgroups, time on treatment and OS curves have not changed substantially from the previous analyses [20,21], although some median values have changed, likely due to the capturing of just a single point on the curve and small patient numbers in some of the subgroups.

Importantly, these clinical benefits were consistent across patient subgroups, including those with poor prognostic characteristics such as brain metastases, age $\geq 65$ years or ECOG PS $\geq 2$, who are often excluded from or underrepresented in randomized clinical trials. Of note, the clinical benefit seen here in patients aged $\geq 65$ years is consistent with that recently reported in a meta-analysis of clinical trial data, which suggested that EGFR TKIs have substantial benefit in elderly patients [22]. Further, it should be noted that prior afatinib treatment did not appear to diminish time on treatment with second-line osimertinib, with patients remaining on second-line 
osimertinib treatment for a median of 15.6 months overall and slightly longer in Asian patients and those with Del19-positive tumors.

These data are in agreement with other studies assessing sequential afatinib and osimertinib. In 37 patients who received osimertinib therapy after first-line afatinib in the LUX-Lung 3, 6 and 7 studies, median time on osimertinib was 20.2 months (95\% CI: 12.8-31.5) and median OS was not reached after a median follow-up of 4.7 years [23]. Recent observational data also support prolonged osimertinib treatment after first-line afatinib [24]. Retrospective analysis of the few patients treated with dacomitinib or afatinib in the Phase III ARCHER-1050 and Phase IIB Lux-Lung 7 trials who went on to receive osimertinib $(n=22$ and $n=20$, respectively), demonstrated that median OS was 36.7 months with sequential dacomitinib and osimertinib, and not reached (3-year OS rate of $\sim 90 \%$ ) with sequential afatinib and osimertinib, respectively $[9,10]$.

The data presented here raise the question of the most appropriate therapeutic strategy: sequential afatinib and osimertinib or first-line osimertinib. OS is clearly a key consideration when selecting first-line treatment. Since the previous analyses of the GioTag study, OS data from the Phase III FLAURA study of first-line osimertinib have been reported; median OS of 38.6 months with osimertinib compared with 31.8 months with first-generation EGFR TKIs (gefitinib or erlotinib) (hazard ratio [HR]: 0.80; 95\% CI: 0.64-1.00; $\mathrm{p}=0.046$ ) [11]. Consequently, osimertinib is increasingly used as a first-line treatment of choice. However, it should be noted that the OS benefit of first-line osimertinib in the 347 Asian patients included in the FLAURA study was less clear with a HR of 1.00 (95\% CI: 0.75-1.32; median OS 37.1 months with osimertinib and 35.8 months with erlotinib/gefitinib) [11,25]. While direct comparisons are limited, not least because the FLAURA study enrolled patients with Del19 or L858R EGFR mutations at diagnosis, whereas the GioTag study only collected data from patients who acquired the T790M mutation after first-line afatinib treatment, the overall OS (37.6 months) reported for the broad, real-world patient population in the GioTag study is similar to that seen in the FLAURA trial. While further work may be needed to further identify patients likely to acquire the T790M mutation, and to identify therapeutic options for T790M-negative patients, it seems that some patient subgroups, such as those with Del19-positive disease and Asian patients, may benefit from a sequential therapy approach.

Further prospective validation is needed to address the question of the optimum therapeutic approach in patients with EGFR mutation-positive NSCLC. The final OS analysis of the Phase III AURA-3 trial, comparing second-line osimertinib with chemotherapy following first-line progression on EGFR TKIs in 419 patients with EGFR mutation-positive NSCLC demonstrated a numerical OS advantage for osimertinib, although this was not statistically significant (median OS: 26.8 vs 22.5 months; HR: 0.87; 95\% CI: $0.67-1.12 ; \mathrm{p}=0.277$ ) [26]. The Phase II APPLE trial (which compares sequential gefitinib/osimertinib vs first-line osimertinib) [27] should also be informative in terms of comparing the OS benefits of different sequential regimens.

As discussed previously [20], the main limitations of the GioTag study were its retrospective nature, lack of a comparator arm and potential for selection bias. The potential for selection bias was minimized as much as possible, for example by including only consecutive patients who fulfilled all of the inclusion criteria and limiting enrollment to a maximum of 15 patients per site. Nevertheless, this may have inadvertently introduced selection bias by either excluding those who died on first-line afatinib or under-representing those who derived long-term benefit from first-line afatinib; data from the LUX-Lung trials estimate these to be approximately 6 and $10-20 \%$ of patients, respectively.

\section{Conclusion}

These final data from the real-world GioTag study confirm those of the previous analyses and demonstrate that sequential afatinib followed by osimertinib is a feasible and effective therapeutic strategy in real-world patients with EGFR mutation-positive NSCLC who develop T790M.

Of note, median OS was over 3.5 years in Asian patients and those with Del19-positive disease, suggesting that sequential use of TKIs could potentially allow these EGFR mutation-positive NSCLC patients to receive long-term, chemotherapy-free treatment. 
Summary points

- The international, observational GioTag study is the first to evaluate outcomes of patients who received first-line afatinib followed by osimertinib; initial and updated analyses showed encouraging results for this sequential approach, particularly for Del19-positive patients and Asian patients. Here, we report findings from the final analysis, including updated time on treatment and overall survival (OS) data.

- Patients had advanced, EGFR mutation-positive (Del19, L858R) non-small-cell lung cancer with T790M-positive disease following first-line afatinib and must have started osimertinib treatment $\geq 10$ months prior to data entry. The primary outcome was time on treatment from initiation of afatinib until discontinuation of osimertinib; the OS analysis was exploratory.

- Overall, in 203 patients analyzed, the median time on EGFR-TKI treatment was 27.7 months (90\% Cl: 26.7-29.9). Median time on treatment was particularly encouraging in patients with Del19-positive disease (median 30.0 months [90\% Cl: 27.6-31.9]) and Asian patients (median 37.1 months [90\% Cl: 28.1-40.3]).

- Clinical benefit was also consistent across patients with poor prognosis; for example, those with Eastern Cooperative Oncology Group performance status $\geq 2$ and stable brain metastases also appeared to derive clinical benefit (median time on treatment 22.2 months for both subgroups).

- Overall median OS was 37.6 months (90\% Cl: $35.5-41.3)$ with a 2-year survival rate of $80 \%$. Particularly encouraging results were again seen for Del19-positive and Asian patients: median OS was 44.8 months $(90 \% \mathrm{Cl}$ : 37.0-57.8) in Asian patients and 41.6 months $(90 \% \mathrm{Cl}: 36.9-45.0)$ in patients with Del19-positive disease.

- In the 31 Asian patients with Del19-positive disease, median time on treatment was 40.0 months $(90 \% \mathrm{Cl}$ : 36.4-45.0) and median OS was 45.7 months (90\% Cl: 38.2-57.8).

- These final data from the real-world GioTag study confirm those of the previous analyses and demonstrate that sequential afatinib followed by osimertinib is a feasible and effective therapeutic strategy in real-world patients with EGFR mutation-positive non-small-cell lung cancer who develop T790M, particularly those with Del19-positive disease and Asian patients.

\section{Supplementary data}

An infographic accompanies this paper and is included at the end of the references section in the PDF version. To view or download this infographic in your browser please click here: www.futuremedicine.com/doi/suppl/10.2217/fon-2020-0740

Author contributions

The authors are fully responsible for all content and editorial decisions, they were also involved at all stages of manuscript development and have approved the final version.

Financial \& competing interests disclosure

This study was supported by Boehringer Ingelheim. MJ Hochmair reports personal fees from Speakers honorarium Boehringer Ingelheim, AstraZeneca and Roche. A Morabito has received honoraria from Boehringer Ingelheim, Roche, AstraZeneca, Pfizer, MSD and Bristol Myers Squibb. D Hao reports research funding and consultancy from Boehringer Ingelheim and Astra Zeneca. RA Soo reports grants and personal fees from Astra Zeneca, personal fees from BMS, Boehringer Ingelheim, Celgene, Lilly, Merck, Novartis, Pfizer, Roche, Taiho and Ignyta. JC-H Yang reports personal fees from Boehringer Ingelheim, Eli Lilly, Roche/Genentech, Chugai, Astellas, MSD, Merck Serono, Pfizer, Novartis, Celgene, Merrimack, Yuhan Pharmaceuticals, Bristol Myers Squibb, Ono Pharmaceuticals, Daiichi Sankyo, AstraZeneca, Hansoh Pharmaceuticals and Takeda Pharmaceuticals. B Halmos reports grants and personal fees from Boehringer Ingelheim, Astra Zeneca, Pfizer, Novartis and Takeda, personal fees from Genentech/Roche, and grants from Merck. A Märten reports employment with Boehringer Ingelheim. T Cufer reports consultancy and honoraria from AstraZeneca, Roche, Pfizer, MSD, Bristol Myers Squibb and Boehringer Ingelheim. C-T Yang and R Gucalp report no competing interests. The authors have no other relevant affiliations or financial involvement with any organization or entity with a financial interest in or financial conflict with the subject matter or materials discussed in the manuscript apart from those disclosed.

Medical writing assistance was provided by J Saunders, of GeoMed, an Ashfield company, part of UDG Healthcare plc and was supported financially by Boehringer Ingelheim.

Ethical conduct of research

The study was carried out in compliance with the protocol, the principles laid down in the Declaration of Helsinki, in accordance with the International Conference on Harmonisation (ICH) Harmonized Tripartite Guideline for Good Clinical Practice, Good Epidemiological Practice, Guidelines for Good Pharmacoepidemiology Practice and relevant sponsor Standard Operating Procedures. 
The study was initiated only after all required legal documentation was reviewed and approved by the respective Institutional Review Board/Independent Ethics Committee and competent authority according to national and international regulations.

Data sharing statement

The datasets generated and analyzed during the study are available from $\mathrm{MH}$ on reasonable request.

Open access

This work is licensed under the Attribution-NonCommercial-NoDerivatives 4.0 Unported License. To view a copy of this license, visit http://creativecommons.org/licenses/by-nc-nd/4.0/

\section{References}

Papers of special note have been highlighted as: $\bullet$ of interest; $\bullet \bullet$ of considerable interest

1. FDA. Tarceva $\mathbb{R}$ (erlotinib), prescribing information. (2016).

https://www.accessdata.fda.gov/drugsatfda_docs/label/2016/021743s025lbl.pdf

2. AstraZeneca. Iressa $®$ (gefitinib), prescribing information. (2015). https://www.azpicentral.com/iressa/iressa.pdf

3. FDA. Gilotrif® (afatinib), prescribing information. (2018).

https://www.accessdata.fda.gov/drugsatfda_docs/label/2018/201292s014lbl.pdf

4. Pfizer. Vizimpro $®$ Tablets (dacomitinib), prescribing information. (2018).

http://labeling.pfizer.com/ShowLabeling.aspx?format=PDF\&id=11019

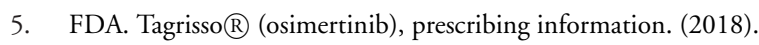

https://www.accessdata.fda.gov/drugsatfda_docs/label/2018/208065s008lbl.pdf

6. Park K, Tan E-H, O’Byrne K et al. Afatinib versus gefitinib as first-line treatment of patients with EGFR mutation-positive non-small-cell lung cancer (LUX-Lung 7): a Phase IIB, open-label, randomised controlled trial. Lancet Oncol. 17(5), 577-589 (2016).

- Randomized Phase IIb trial (LUX-Lung 7) which demonstrates improved progression-free survival (PFS) and time to treatment failure (TTF) with afatinib versus gefitinib in patients with EGFR mutation-positive non-small-cell lung cancer (NSCLC).

7. Wu YL, Cheng Y, Zhou X et al. Dacomitinib versus gefitinib as first-line treatment for patients with $E G F R$-mutation-positive non-small-cell lung cancer (ARCHER 1050): a randomised, open-label, phase 3 trial. Lancet Oncol. 18(11), 1454-1466 (2017).

-. Randomized Phase III ARCHER 1050 trial which demonstrates improved PFS with dacomitinib versus gefitinib in patients with EGFR mutation-positive NSCLC.

8. Soria JC, Ohe Y, Vansteenkiste J et al. Osimertinib in untreated EGFR-mutated advanced non-small-cell lung cancer. N. Engl. J. Med. 378(2), 113-125 (2018).

-• Randomized Phase III FLAURA trial demonstrating improved PFS with osimertinib versus gefitinib or erlotinib in patients with EGFR mutation-positive NSCLC.

9. Mok TS, Cheng Y, Zhou X et al. Improvement in overall survival in a randomized study that compared dacomitinib with gefitinib in patients with advanced non-small-cell lung cancer and EGFR-activating mutations. J. Clin. Oncol. 36(22), 2244-2250 (2018).

-• Mature overall survival (OS) data from ARCHER 1050 which demonstrates significant $O S$ benefit with dacomitinib versus gefitinib in patients with EGFR mutation-positive NSCLC.

10. Paz-Ares L, Tan E-H, O’Byrne $\mathrm{K}$ et al. Afatinib versus gefitinib in patients with $E G F R$ mutation-positive advanced non-small-cell lung cancer: overall survival data from the Phase IIb LUX-Lung 7 trial. Ann. Oncol. 28(2), 270-277 (2017).

11. Ramalingam SS, Vansteenkiste J, Planchard D et al. Overall survival with osimertinib in untreated, EGFR-mutated advanced NSCLC. N. Engl. J. Med. 382(1), 41-50 (2020).

-• Updated data from FLAURA demonstrating significant OS benefit with osimertinib versus gefitinib or erlotinib.

12. Arcila ME, Oxnard GR, Nafa K et al. Rebiopsy of lung cancer patients with acquired resistance to EGFR inhibitors and enhanced detection of the T790M mutation using a locked nucleic acid-based assay. Clin. Cancer Res. 17(5), 1169-1180 (2011).

13. Sequist LV, Waltman BA, Dias-Santagata D et al. Genotypic and histological evolution of lung cancers acquiring resistance to EGFR inhibitors. Sci. Transl. Med. 3(75), 75ra26 (2011).

14. Yang JC, Ahn M-J, Kim D-W et al. Osimertinib in pretreated T790M-positive advanced non-small-cell lung cancer: AURA study Phase II extension component. J. Clin. Oncol. 35(12), 1288-1296 (2017).

15. Hochmair MJ, Buder A, Schwab S et al. Liquid-biopsy-based identification of EGFR T790M mutation-mediated resistance to afatinib treatment in patients with advanced EGFR mutation-positive NSCLC, and subsequent response to osimertinib. Target Oncol. 14(1), 75-83 (2019).

16. Jenkins S, Yang JC-H, Ramalingam SS et al. Plasma ctDNA analysis for detection of the EGFR T790M mutation in patients with advanced non-small-cell lung cancer. J. Thorac. Oncol. 12(7), 1061-1070 (2017). 
17. Mok TS, Wu Y-L, Ahn M-J et al. Osimertinib or platinum-pemetrexed in EGFR T790M-positive lung cancer. N. Engl. J. Med. 376(7), 629-640 (2017).

- Phase III AURA3 study demonstrating significant PFS benefit with osimertinib versus chemotherapy in patients with T790M-positive tumors following progression of erlotinib, gefitinib or afatinib.

18. Oxnard GR, Hu Y, Mileham KF et al. Assessment of resistance mechanisms and clinical implications in patients with EGFR T790M-positive lung cancer and acquired resistance to osimertinib. JAMA Oncol. 4(11), 1527-1534 (2018).

19. Niederst MJ, Hu H, Mulvey HE et al. The allelic context of the C797S mutation acquired upon treatment with third-generation EGFR inhibitors impacts sensitivity to subsequent treatment strategies. Clin. Cancer Res. 21(17), 3924-3933 (2015).

20. Hochmair MJ, Morabito A, Hao D et al. Sequential treatment with afatinib and osimertinib in patients with EGFR mutation-positive non-small-cell lung cancer: an observational study. Future Oncol. 14(27), 2861-2874 (2018).

- Initial results from the GioTag study demonstrating prolonged time on treatment with sequential afatinib and osimertinib in patients with EGFR mutation-positive NSCLC.

21. Hochmair MJ, Morabito A, Hao D et al. Sequential afatinib and osimertinib in patients with EGFR mutation-positive non-small-cell lung cancer: updated analysis of the observational GioTag study. Future Oncol. 15(25), 2905-2914 (2019).

- Updated, interim results from the GioTag study which demonstrates prolonged OS with sequential afatinib and osimertinib in patients with EGFR mutation-positive NSCLC.

22. Roviello G, Zanotti L, Cappelletti MR et al. Are EGFR tyrosine kinase inhibitors effective in elderly patients with $E G F R$-mutated non-small-cell lung cancer? Clin. Exp. Med. 18(1), 15-20 (2018).

23. Park K, Bennouna J, Boyer M et al. Sequencing of therapy following first-line afatinib in patients with EGFR mutation-positive non-small-cell lung cancer. Lung Cancer 132, 126-131 (2019).

24. Tamiya M, Tamiya A, Suzuki $\mathrm{H}$ et al. Which is better EGFR-TKI followed by osimertinib: afatinib or gefitinib/erlotinib? Anticancer Res. 39(7), 3923-3929 (2019).

25. Nogami N, Ramalingam SS, Imamura F et al. PS-1 Osimertinib as first-line therapy for EGFRm advanced NSCLC (FLAURA): final OS in Japanese subset. Presented at: The 60th Annual Meeting of the Japanese Lung Cancer Society. (2019). https://www.haigan.gr.jp/journal/am/2019a/19a_pdsy0000PS-1.html

26. Wu Y, Mok TSK, Han J-Y et al. Overall survival (OS) from the AURA3 Phase III study: osimertinib vs platinum-pemetrexed (plt-pem) in patients (pts) with EGFR T790M advanced non-small cell lung cancer (NSCLC) and progression on a prior EGFR-tyrosine kinase inhibitor (TKI). Ann. Oncol. 30(Suppl. 9), ix157-ix181 (2019).

27. Remon J, Caramella C, Jovelet C et al. Osimertinib benefit in EGFR-mutant NSCLC patients with T790M-mutation detected by circulating tumour DNA. Ann. Oncol. 28(4), 784-790 (2017). 


\section{GioTag study: concept}

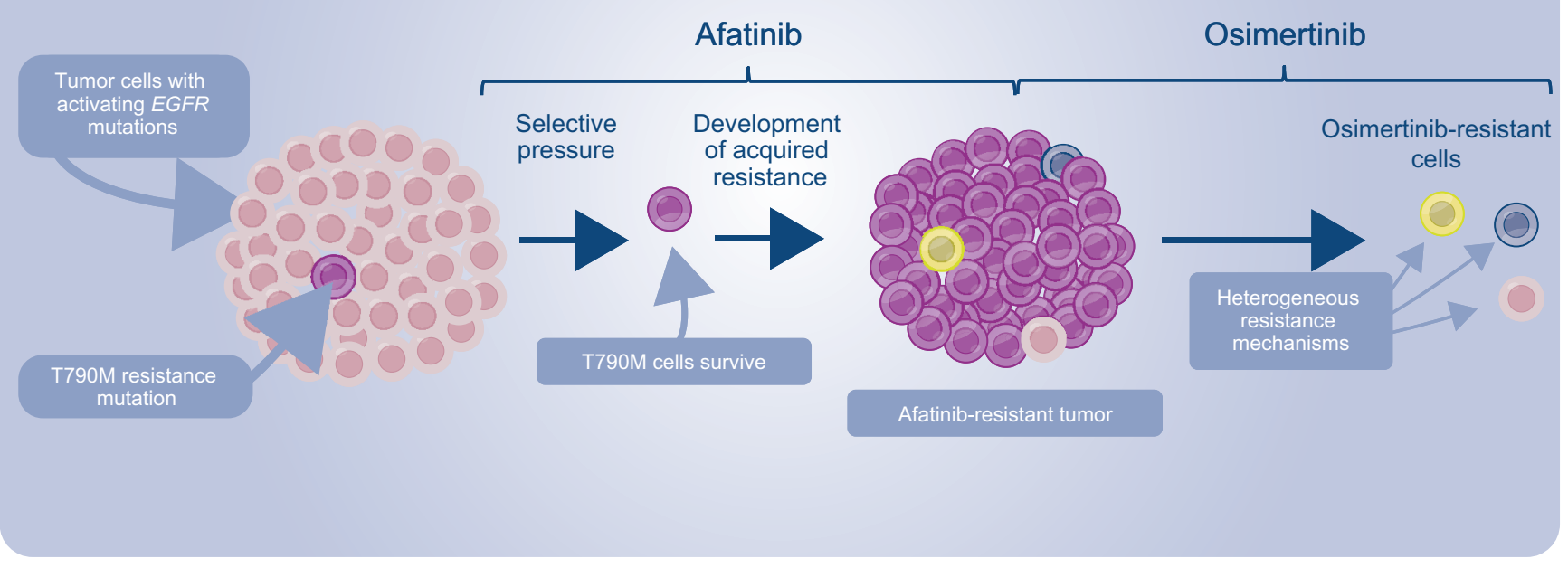

\section{GioTag: design and results}

Patients ( $n=203$ ): received first-line afatinib followed by osimertinib after developing T790M-mediated resistance

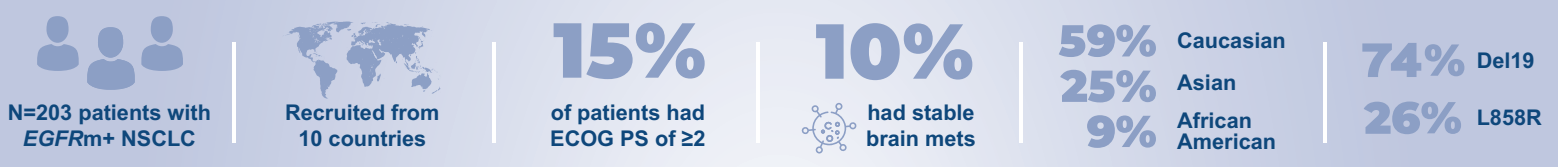

Median time on treatment (months)

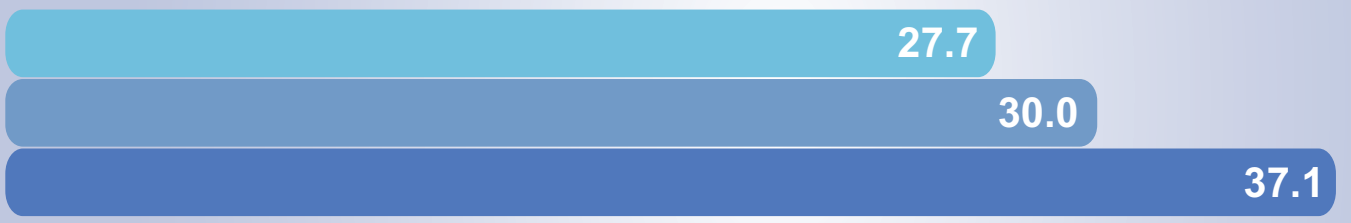

Median OS (months)

37.6

41.6

44.8

All patients $(n=203)$

Patients with Del19+ tumors $(n=149)$

Asian patients $(n=50)$ 